\title{
Characterizing Self-Assembled Monolayer Breakdown in Area-Selective Atomic Layer Deposition
}

Tzu-Ling Liu ${ }^{\ddagger 1}$, Li Zeng ${ }^{\ddagger 2}$, Katie L. Nardi ${ }^{3}$, Dennis M. Hausmann ${ }^{3}$, and Stacey F. Bent ${ }^{1,2^{*}}$

${ }^{1}$ Department of Materials Science and Engineering, Stanford University, 496 Lomita Mall, Stanford, CA USA 94305.

${ }^{2}$ Department of Chemical Engineering, Stanford University, 443 Via Ortega, Stanford, CA USA 94305.

${ }^{3}$ Lam Research Corporation, 4650 Cushing Parkway, Fremont, CA USA 94538.

$\$$ These authors contributed equally to this work.

*sbent@stanford.edu
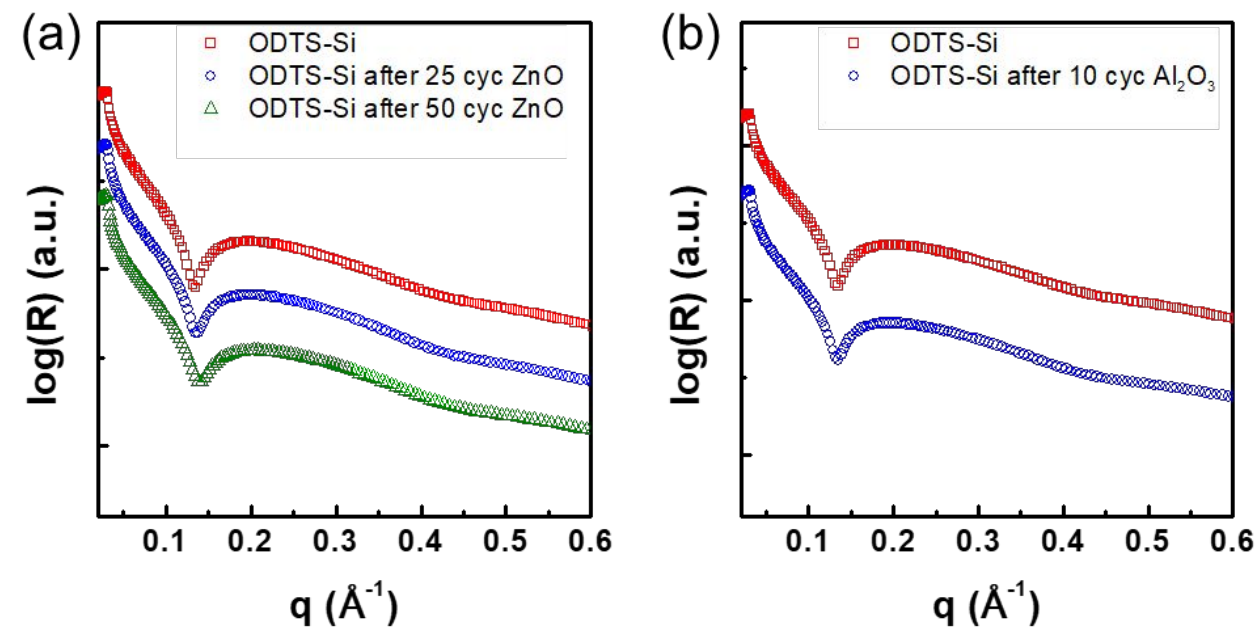

Figure S1. (a) XRR measurements (symbols) of ODTS-Si without any ALD and with 25 and 50 cycles $\mathrm{ZnO}$ ALD. (b) XRR measurements (symbols) of ODTS-Si without any ALD and with 10 cycles $\mathrm{Al}_{2} \mathrm{O}_{3}$ ALD. 


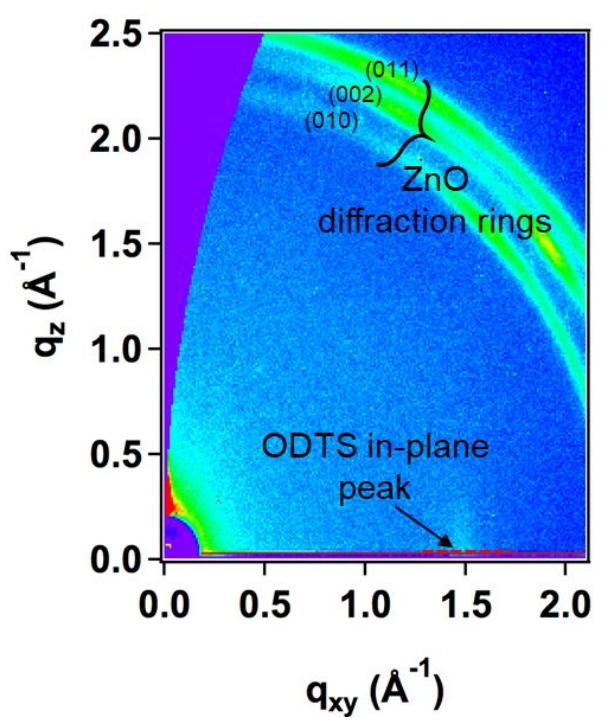

Figure S2. GIWAXS pattern of ODTS-sapphire after 100 cycles ZnO ALD. The red dashed line indicates how the line profile of the diffraction peak of ODTS SAM is extracted for display in Figure 8.

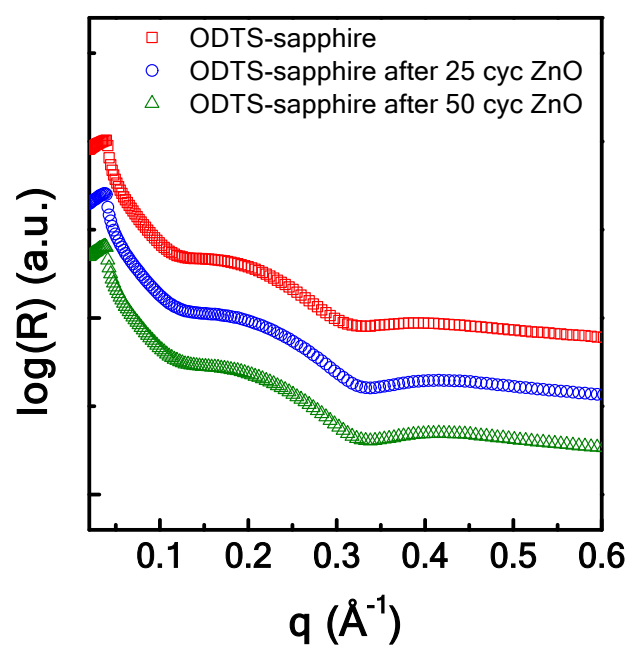

Figure S3. (a) XRR measurements (symbols) of ODTS-sapphire without any ALD and with 25 and 50 cycles $\mathrm{ZnO}$ ALD. 

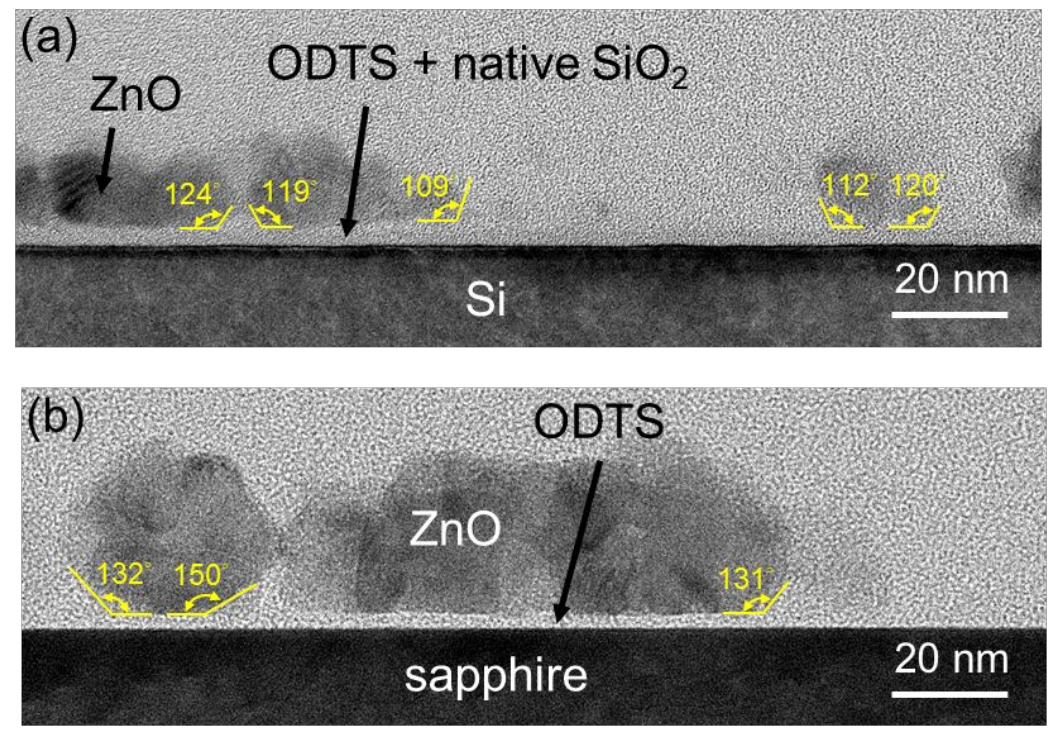

Figure S4. TEM images (taken from Figure 6 and 10) of (a) ODTS-Si and (b) ODTS-sapphire after 100 cycles of $\mathrm{ZnO} \mathrm{ALD}$ with the contact angles of $\mathrm{ZnO}$ nanoparticles marked. 